\title{
Glass Transition and Enthalpy Relaxation of Sucrose - Glucose Syrup Solid System
}

\author{
Liu, Y. ${ }^{\text {a }}, \underline{\text { Zhou, }}$ W. $^{\text {a }}{ }^{*}$, Bhandari, B. ${ }^{\mathrm{b}}$ \\ ${ }^{a}$ Food Science \& Technology Programme \\ Department of Chemistry, National University of Singapore \\ 3 Science Drive 3, Singapore 117543 \\ ${ }^{\mathrm{b}}$ School of Land and Food Sciences, The University of Queensland \\ Brisbane QLD 4072, Australia \\ * Corresponding author. Email: chmzwb@nus.edu.sg
}

\begin{abstract}
Glass transition and enthalpy relaxation of sucrose, glucose syrup solid (GSS) and their mixtures $(75 / 25$. 50/50, 25/75 sucrose/GSS) have been investigated in terms of CouchmanKarasz equation and Kohlrausch-Williams-Watts (KWW) model parameters using differential scanning calorimetry (DSC) data. Addition of GSS up to $50 \%$ did not significantly increase the glass transition temperature of the mixtures compared to the glass transition temperature of sucrose. Addition of GSS into sucrose increased the mean relaxation time $\tau$ dramatically at all aging temperatures. In general, the more GSS added, the longer the mean relaxation time of the mixture, at the aging temperature that has the same level below its glass transition temperature. Addition of GSS into sucrose up to $50 \%$ did not change the relaxation susceptibility significantly. Addition of GSS into sucrose increased the relaxation time distribution spectrum and generally decreased the $\beta$ values.
\end{abstract}

Keywords: sucrose; glucose syrup solid; glass transition; enthalpy relaxation; DSC

Resulted from food processing such as extrusion, thermal treatments, and dehydration, many processed solid food materials are in amorphous state, where glass transition and structural relaxation are the two main state transitions. Both of them relate to the molecular mobility and therefore the physical properties, which induce quality changes of the amorphous food products during storage. Sucrose and glucose syrup are among the commonly and widely used ingredients in the food industry. Through processing, they exist in amorphous state in both rapidly dried and frozen foods. Therefore, the knowledge of glass transition and enthalpy relaxation of sucrose-glucose syrup system is important to asurring the quality of foods containing these two ingredients during storage.

GSS with higher $T_{g}$ added into sucrose was expected to function as an anti-palsticizer to raise the $\mathrm{T}_{\mathrm{g}}$ of the mixtures. However it was found that addition of GSS up to $50 \%$ did not have a significant anti-palsticizing effect (Figure 1). Similar behaviour by other carobohydrate antiplasticizers were also found in other studies. The $T_{g}$ values of the mixtures were compared to those predicted using Couchman-Karasz equation (Figure 1). The deviations between the experimental values and predicted values suggested that the mixing be non-ideal in the mixtures. The relaxation behavior of all the samples was well-predicted by KWW equation. Addition of GSS into sucrose retarded the enthalpy relaxtion of the mixtures at all aging temperatures, which was indicated by the increased $\tau$ values (mean relaxation time) of KWW equation (Figure 2). This information could be very useful to minimizing the quality changes of the relavent products during storage. 


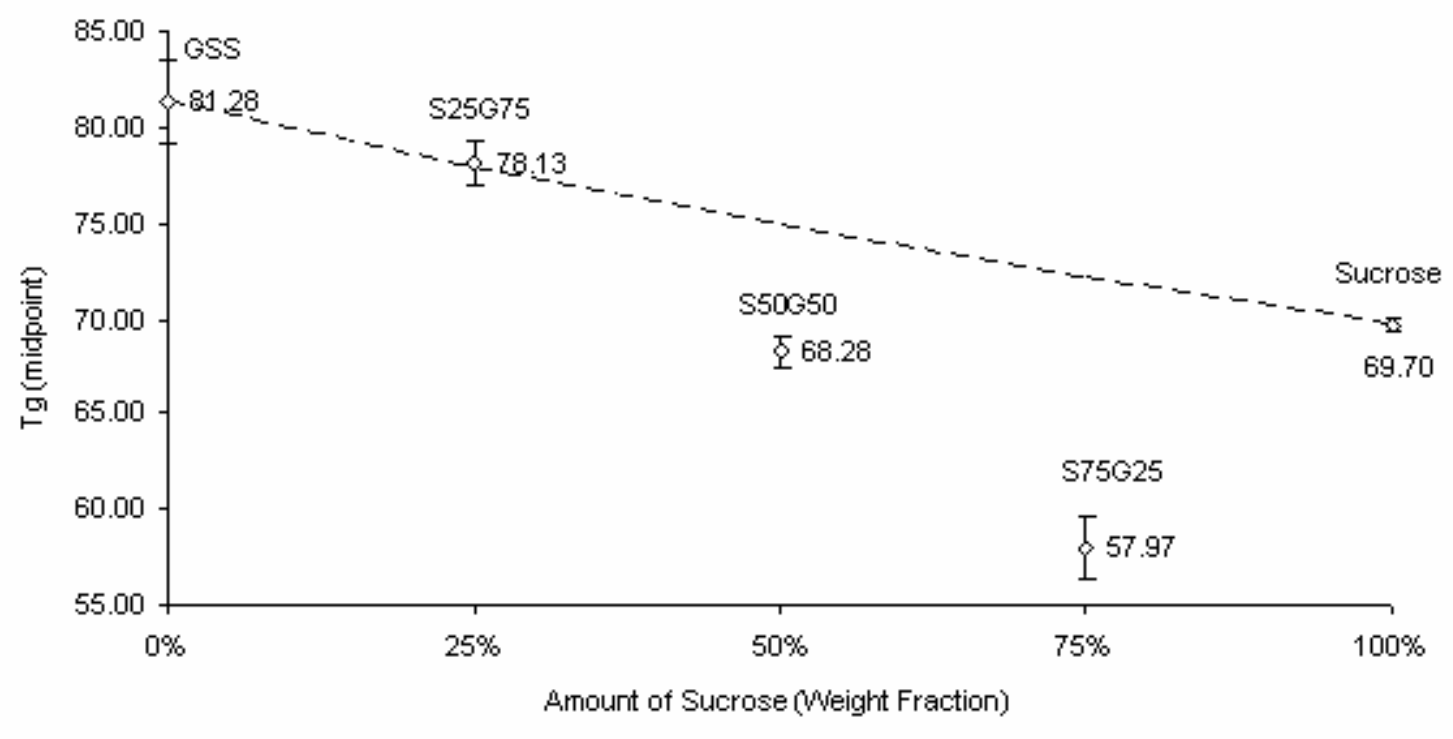

Figure 1: Comparison between the experimental and predicted glass transition temperatures (midpoint). The prediction was based on Coucheman-Karasz equation using the experimental values $\left(\mathrm{T}_{\mathrm{g}}\right.$ midpoint and $\left.\Delta \mathrm{C}_{\mathrm{p}}\right)$ of sucrose and GSS.

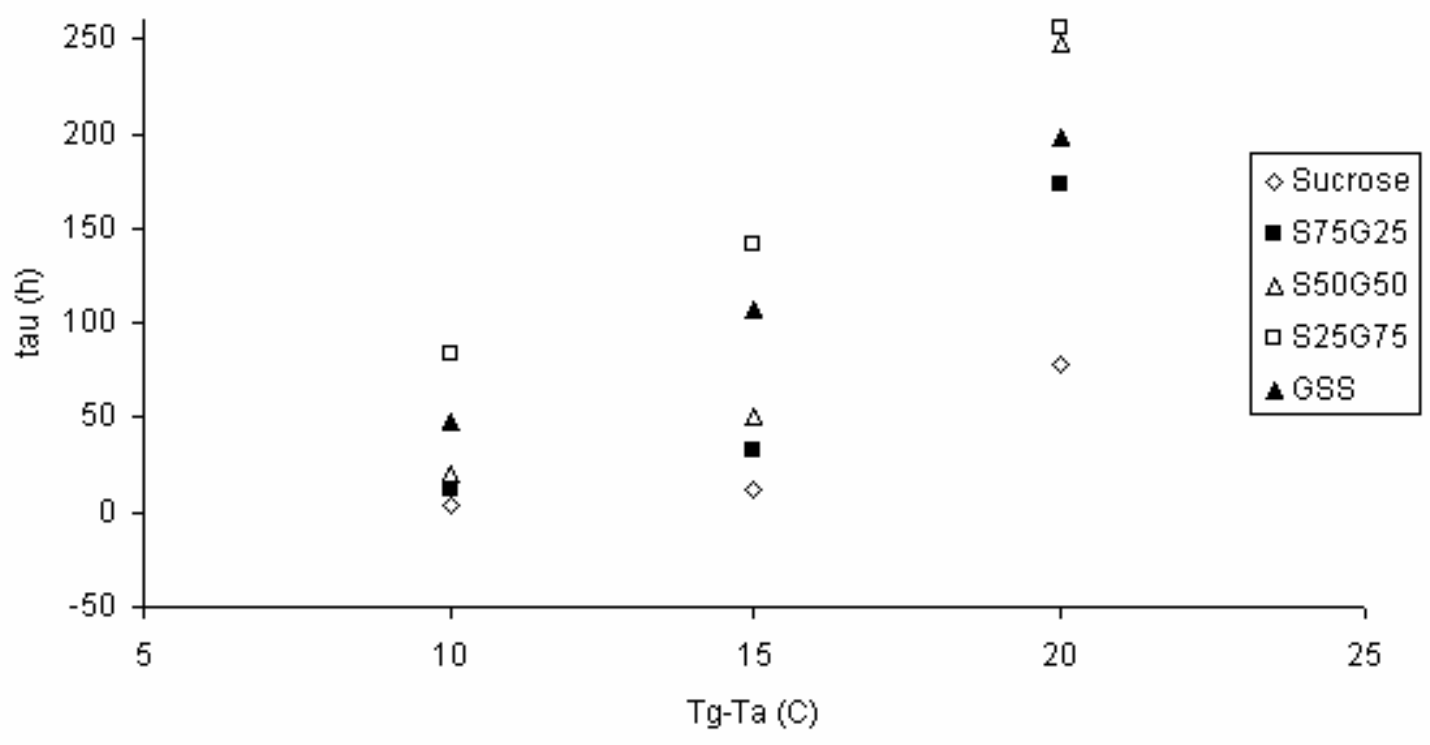

Figure 2: Plot of $\tau$ vs. $\mathrm{T}_{\mathrm{g}}-\mathrm{T}_{\mathrm{a}}$. The $\tau$ values were calculated based on Kohlrausch-WilliamsWatts (KWW) equation using the relaxation enthalpy values. 